\title{
Les autolysines des bactéries lactiques
}

\author{
MP Chapot-Chartier
}

Unité de recherches de biochimie et structure des protéines, Inra, domaine de Vilvert, 78352 Jouy-en-Josas Cedex, France

\begin{abstract}
Résumé - Les autolysines sont définies comme des enzymes bactériennes endogènes capables d'hydrolyser le peptidoglycane, qui est le constituant majeur et essentiel de la paroi. Leur action peut donc induire la lyse bactérienne. Un certain nombre d'autolysines ont été caractérisées au niveau moléculaire chez différentes bactéries. Le rôle physiologique de ces enzymes, potentiellement dangereuses pour la cellule, n'est pas complètement élucidé. Elles jouent un rôle dans la séparation des cellules en fin de division cellulaire, dans le renouvellement du peptidoglycane, la formation de flagelles ou encore durant la sporulation. Dans le cas des bactéries lactiques utilisées dans la fabrication des produits laitiers fermentés, l'autolyse des cellules, en permettant de libérer leur contenu enzymatique intracytoplasmique, apparaît comme un moyen d'obtenir un développement plus rapide des qualités organoleptiques, une intensification de certains arômes ou encore une diminution de l'amertume. Les données actuellement disponibles sur le système autolytique des bactéries lactiques sont encore limitées et concernent essentiellement les lactocoques et les lactobacilles. Les propriétés autolytiques de différentes souches ont été étudiées en milieu de culture contenant différents sucres ainsi qu'en solution tamponnée. Par ailleurs, la technique d'électrophorèse SDS-PAGE renaturante consistant à inclure un substrat d'autolysine tel que des bactéries autoclavées ou des préparations de parois dans le gel d'acrylamide, permet de mettre en évidence différentes activités peptidoglycane hydrolases chez les lactocoques et les lactobacilles. L'activité autolysine majeure présente chez Lactococcus lactis subsp cremoris ainsi que chez Lactobacillus helveticus et Lactobacillus acidophilus a été identifiée comme étant de type muramidase. Récemment, le gène codant pour cette enzyme a été cloné et séquencé chez $L$ lactis et il a été montré que cette enzyme est impliquée dans la séparation des cellules lors de la division cellulaire. L'impact de la lyse des bactéries du levain sur l'affinage a été mis en évidence dans le cas de quelques souches de lactocoques dans des fromages à pâte pressée. La lyse précoce des bactéries est corrélée avec un niveau de protéolyse plus élevé des caséines et un niveau d'amertume plus faible. Le développement des connaissances concernant le système autolytique des bactéries lactiques, la caractérisation moléculaire des enzymes et l'étude de la régulation de leur expression devrait fournir les outils nécessaires à l'obtention de souches qui se lysent précocement au cours de l'affinage.
\end{abstract}

autolysine / lyse / peptidoglycane / bactérie lactique / affinage du fromage

Summary - Autolysins of lactic acid bacteria. Autolysins are defined as endogenous bacterial enzymes able to hydrolyze peptidoglycan which is the main and shape-maintaining component of the cell wall and thus their action can induce bacteriolysis. A number of autolysins have been characterized at the molecular level in different bacteria. The physiological role of these potentially lethal enzymes is not fully understood. Their play a role in cell separation at the end of cell division, in cell wall 
turn-over, in flagella formation or in sporulation. In the particular case of lactic acid bacteria which are widely used in dairy industry, cell autolysis allowing the release of intracellular enzymatic content is expected to result in more rapid development of organoleptic properties, aroma intensification as well as bitterness decrease. Knowledge of the autolytic system from lactic acid bacteria is still incomplete and concerns mainly lactococci and lactobacilli. Autolytic properties of different strains were studied in culture medium containing different sugar concentrations and in buffered solutions. Renaturing electrophoresis which consists in SDS-PAGE of a polyacrylamide gel containing an autolysin substrate such as inactivated bacteria or cell wall preparations, allows to detect different peptidoglycan hydrolase activities in lactococi and lactobacilli. The major autolysin activity present in Lactococcus lactis subsp cremoris as well as in Lactobacillus helveticus or Lactobacillus acidophilus was identified as a muramidase. Recently, the gene encoding this muramidase was cloned and sequenced and it was shown to be involved in cell separation at the end of cell division. The impact of starter lactic acid bacteria lysis on cheese ripening was evidenced in the case of a few strains of lactococci in pressed-type cheese. Early lysis of bacteria was correlated with higher level of proteolysis and lower level of bitterness. Further studies concerning the autolytic system of lactic acid bacteria, molecular characterization of the enzymes and regulation of the expression of their activity should provide the tools necessary to obtain strains which lyse early during ripening.

autolysin / lysis / peptidoglycan / lactic acid bacteria / cheese ripening

\section{INTRODUCTION}

De nombreuses bactéries produisent des enzymes qui sont capables d'hydrolyser des liaisons du peptidoglycane de leur propre paroi. Le peptidoglycane étant le constituant majeur de la paroi et essentiel pour le maintien de l'intégrité structurale de la cellule, l'action de ces enzymes peut conduire à la lyse de la bactérie. Dans ce cas, ces peptidoglycane hydrolases sont également appelées autolysines. L'autolyse d'une culture bactérienne peut être observée dans des conditions qui provoquent un blocage de la synthèse du peptidoglycane telles que des conditions de privation nutritionnelle ou la présence de certains antibiotiques (Shockman et Höltje, 1994 pour revue). La lyse paraît alors résulter d'une action incontrôlée des autolysines. Par ailleurs, le fait que ces enzymes potentiellement dangereuses pour la cellule sont largement répandues chez les bactéries et sont présentes dans des cultures en cours de division, a conduit à l'idée que les autolysines jouent un rôle physiologique important et qu'elles sont impliquées dans la croissance bactérienne et l'assemblage du peptidoglycane (Rogers et al, 1980 ; Doyle et Koch, 1987 ; Höltje et Tuomanen, 1991).

Dans le cas des bactéries lactiques, le phénomène d'autolyse présente un intérêt particulier quant à son utilisation potentielle dans la fabrication des produits laitiers fermentés. En effet, le contenu enzymatique intracellulaire des bactéries lactiques du levain joue un rôle important dans le développement de la flaveur et de la texture des fromages (Visser, 1977 ; Olson, 1990 ; Visser, 1993). Parmi ces enzymes, les mieux caractérisées sont les peptidases qui libèrent des petits peptides et des acides aminés libres qui sont des précurseurs d'arômes. L'action des peptidases contribue également à diminuer l'amertume. Actuellement, les connaissances acquises sur le système peptidasique des lactocoques suggèrent que toutes les peptidases sont localisées à l'intérieur du cytoplasme bactérien (Tan et al, 1992 ; Monnet et al, 1993). Les études effectuées sur les autres bactéries lactiques, streptocoques thermophiles ou lactobacilles, bien que moins approfondies (Pritchard et Coolbear, 1993), indiquent qu'elles possèdent un équipement peptidasique analogue. En conséquence, la lyse des bactéries condui- 
sant à la libération des peptidases dans le caillé fromager devrait permettre d'intensifier leur action en facilitant l'accès à leur substrat. De même, l'action d'autres enzymes intracytoplasmiques intervenant dans le développement de la flaveur telles que des estérases, des lipases ou des enzymes de dégradation des acides aminés pourrait être amplifiée.

L'induction contrôlée de l'autolyse des bactéries du levain apparaît ainsi comme une voie prometteuse pour accélérer l'affinage, intensifier la production de certains arômes à temps d'affinage égal ou encore limiter la production d'amertume dans les produits laitiers fermentés. Une connaissance plus approfondie du système autolytique des bactéries lactiques apparaît nécessaire afin de contrôler le processus d'autolyse.

Avant de décrire les données actuellement disponibles sur le système autolytique des bactéries lactiques, nous présentons d'abord une brève revue concernant les caractéristiques des autolysines les mieux caractérisées chez quelques bactéries autres que les bactéries lactiques. Nous décrivons enfin les résultats qui montrent l'influence de la lyse des bactéries du levain sur l'affinage.

\section{AUTOLYSINES BACTÉRIENNES}

La paroi des bactéries à Gram positif est constituée d'un assemblage de différents polymères : peptidoglycane, acides teichoïques, sucres, protéines. Le peptidoglycane est le constituant majeur de la paroi ( 50 à $80 \%$ en poids sec) et essentiel à son intégrité structurale. Il est constitué de chaînes linéaires de polysaccharides composées d'un enchaînement d'acide $\mathrm{N}$-acétyl-muramique (NAc-Mur) et de $\mathrm{N}$-acétylglucosamine (NAc-Glc) et ces chaînes sont liées entre elles par de courtes chaînes peptidiques (Schleifer et Kandler, 1972 ;
Shockman et Barrett, 1983). Différentes classes d'autolysines peuvent être définies selon leur spécificité d'hydrolyse du peptidoglycane (Ghuysen, 1968 ; Shockman et Barrett, 1983) (fig 1):

- les N-acétyimuramidases (ou lysozymes) qui hydrolysent la liaison $\beta 1-4$ entre acide $N$ acétyl-muramique et $\mathrm{N}$-acétylglucosamine ; - les $\beta$-N-acétylglucosaminidases qui hydrolysent la liaison $\beta 1-4$ entre $\mathrm{N}$-acétylglucosamine et acide $\mathrm{N}$-acétylmuramique :

- les N-acétylmuramyl-L-alanine amidases (amidases) qui hydrolysent la liaison entre le groupement lactyl de l'acide muramique et le groupement $\alpha$-aminé de la $L$-alanine, premier acide aminé de la chaîne peptidique ; - les peptidases qui hydrolysent les chaînes peptidiques principales ou les ponts interchaînes.

Une espèce bactérienne donnée peut produire une ou plusieurs autolysines, possédant une spécificité d'hydrolyse du peptidoglycane identique ou non. Un certain nombre d'autolysines ont été caractérisées au niveau moléculaire chez différentes bactéries à Gram positif telles que Bacillus subtilis, Streptococcus pneumoniae, Staphylococcus aureus, Enterococcus hirae ou des bactéries à Gram négatif en particulier Escherichia coli. Une revue récente et complète de Shockman et Höltje (1994) fait le point sur les connaissances actuellement disponibles sur les peptidoglycane hydrolases microbiennes. Nous nous limiterons ici à présenter les données relatives aux autolysines les mieux caractérisées chez les bactéries à Gram positif.

Un certain nombre d'autolysines ont été purifiées à homogénéité. Néanmoins, la purification de ces enzymes s'est révélée être une tâche relativement difficile car elles sont en général peu abondantes dans la cellule et fortement liées à la paroi. Plus récemment, les gènes codant pour certaines d'entre elles ont été clonés et séquencés. Le clonage a été réalisé soit en utilisant des 


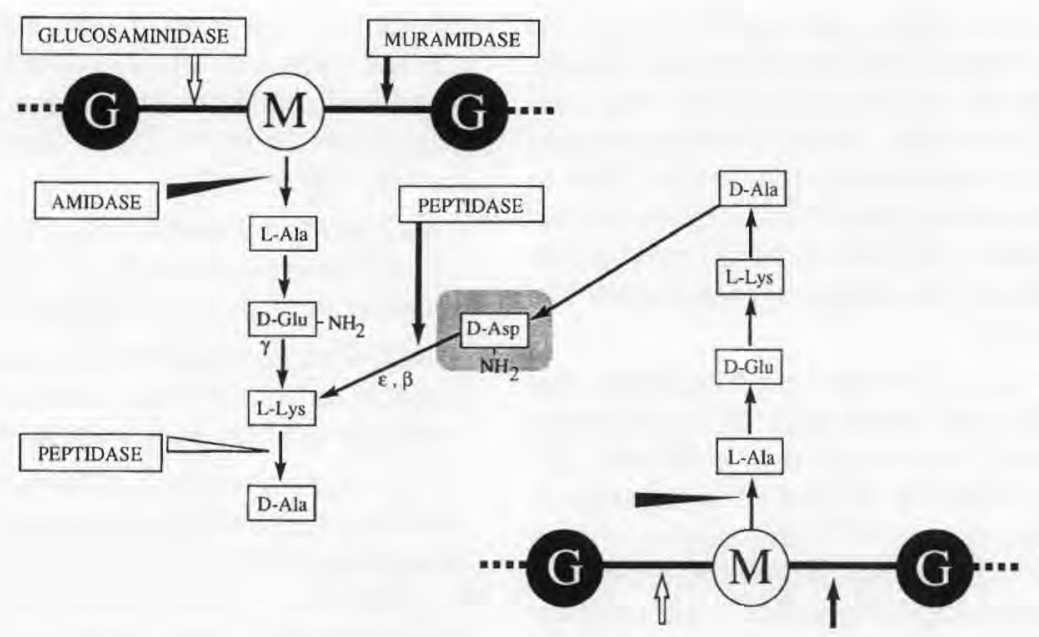

Fig 1. Structure du peptidoglycane des lactocoques et lactobacilles et spécificité des autolysines. Le peptidoglycane est formé de chaînes de polysaccharides composées de $\mathrm{N}$-acétylglucosamine $(\mathrm{G})$ et d'acide $N$-acétylmuramique (M) liés par des liaisons glycosidiques $\beta-1,4$. Sur ces chaînes de polysaccharides sont fixées de manière covalente des chaînes peptidiques composées de L-Ala, D-Glu, L-Lys et D-Ala, dont certaines sont pontées entre elles par l'intermédiaire d'un D-Asp dans le cas des lactocoques et lactobacilles. Les sites de coupure des différentes classes d'autolysines sont indiquées sur le schéma.

Structure of the peptidoglycan of lactococci and lactobacilli and specificity of the autolysins. Peptidoglycan is formed by polysaccharide chains composed of $\mathrm{N}$-acetyl-glucosamine (G) and $\mathrm{N}$-acetylmuramic acid $(N)$ linked by $\beta-1,4$ glycosidic bonds. On these chains are covalently linked peptide chains composed of L-Ala, D-Glu, L-Lys and D-Ala, and part of them are linked by a bridge consisting of D-Asp in the case of lactococci and lactobacilli. The bonds hydrolysed by the different classes of autolysins are indicated.

sondes oligonucléotidiques déduites des séquences en acides aminés de la protéine purifiée, soit en effectuant un criblage d'activité d'une banque d'ADN construite chez $E$ coli. Les propriétés des autolysines les mieux caractérisées chez les bactéries à Gram positif sont résumées dans le tableau I et discutées ci-dessous.

\section{Structure des autolysines}

Les enzymes caractérisées possèdent des masses moléculaires très variables (tableau I). En revanche, elles paraissent avoir en commun une structure composée de deux domaines, l'un contenant le site catalytique et le second contenant le site de liaison à la paroi. Cette organisation modulaire a été proposée initialement sur la base des similarités de séquence existant entre l'amidase majeure de $S$ pneumoniae et les enzymes lytiques, amidases ou muramidases, provenant de bactériophages qui infectent cette bactérie (Garcia et al, 1988a). Elle a été confirmée par la possibilité de construire des enzymes chimériques en choisissant d'une part la spécificité d'hydrolyse et d'autre part la spécificité de liaison des parois (Lopez et al, 1992). Le domaine de liaison et reconnaissance de la paroi peut être identifié par la présence de séquences répétées de longueur variable ( 20 acides aminés ou plus) et en 
Tableau I. Principales autolysines bien caractérisées chez les bactéries à Gram positif. Main autolysins characterized in Gram-positive bacteria.

\begin{tabular}{|c|c|c|c|c|c|c|}
\hline $\begin{array}{l}\text { Organisme } \\
\text { et enzyme }\end{array}$ & $\begin{array}{l}\text { Gène } \\
\text { cloné }\end{array}$ & $\begin{array}{l}M M \\
(k D a)\end{array}$ & $\begin{array}{l}\text { Séquence } \\
\text { signal }\end{array}$ & $\begin{array}{l}\text { Structure } \\
\text { Similarité de séquence }\end{array}$ & Mutation du gène & Références \\
\hline
\end{tabular}

\section{Bacillus subtilis}

Amidase CwIB

(LytC)

oui

52,6

oui

(24 a.a) 3 séquences répétées (57 aa)

C-terminal :

domaine catalytique

\section{Amidase CwIA}

oui

27,1

Amidase CwIC

oui

LytD
95,6

oui

(27 aa) -terminal :

2 séquences répétées
Effet sur :

- diminution activité amidase

- résistance accrue à la lyse

Kuroda et Sekiguchi (1991)

Margot et Karamata (1992)

Lazarevic et al (1992)

- turn-over du peptidoglycane

- motilité flagellaire

Pas d'effet sur :

- vitesse de croissance

- morphologie des cellules

- séparation des cellules

- transformabilité

- sporulation

Pas d'effet sur :

- croissance

- division cellulaire

- transformabilité

- Induction au cours

de la sporulation

Kuroda et Sekiguchi (1990)

Forster (1991)

Pas d'effet sur :

- lyse des cellules mères

- germination des spores

- caractéristiques des spores

Pas d'effet sur :

Margot et al (1994)

- croissance

- mobilité flagellaire

- séparation des cellules

- autolyse

- turn-over du peptidoglycane

- transformabilité

- sporulation 
Tableau I. Suite.

\begin{tabular}{|c|c|c|c|c|c|c|}
\hline $\begin{array}{l}\text { Organisme } \\
\text { et enzyme }\end{array}$ & $\begin{array}{l}\text { Gène } \\
\text { cloné }\end{array}$ & $\begin{array}{l}M M \\
(k D a)\end{array}$ & $\begin{array}{l}\text { Séquence } \\
\text { signal }\end{array}$ & $\begin{array}{l}\text { Structure } \\
\text { Similarité de séquence }\end{array}$ & Mutation du gène & Références \\
\hline \multicolumn{7}{|c|}{ Streptococcus pneumoniae } \\
\hline Amidase LytA & oui & 36,5 & non & $\begin{array}{l}\text { N-terminal: } \\
\text { domaine catalytique } \\
\text { C-terminal : } \\
\text { - } 6 \text { séquences répétées ( } 21 \text { aa) } \\
\text { - homologies avec les séquences } \\
\text { des lysines de phages } \\
\text { de } S \text { pneumoniae }\end{array}$ & $\begin{array}{l}\text { Effet sur: } \\
\text { - séparation des cellules } \\
\text { - pas d'autolyse en } \\
\text { phase stationnaire } \\
\text { - réponse aux } \beta \text {-lactames } \\
\text { Pas d'effet sur: } \\
\text { - vitesse de croissance } \\
\text { - transformabilité }\end{array}$ & $\begin{array}{l}\text { Garcia et al (1985) } \\
\text { Garcia et al (1986) } \\
\text { Sanchez-Puelles et al (1986) }\end{array}$ \\
\hline
\end{tabular}

\section{Glusaminidase}

non

64

\section{Enterococcus hirae}

Muramidase-1

non

130

(zymogène)

87

(active)

Muraminidase-2

oui

70,7

53,8

\section{Staphylococcus aureus}

Amidase LytA non

oui $\mathrm{N}$-terminal :

(49 aa) domaine catalytique

C-terminal :

- 6 séquences répétées (45 aa)

- homologie avec autolysine

de $S$ faecalis

C-terminal :

homologie avec la lysostaphine

(endopeptidase) de Staphylococcus

simulans

\section{Garcia et al (1989)}

Kawamura et Shockman

(1983)

Chu et al (1992)
Enzyme de prophage
Jayaswal et al (1990)
Wang et al (1991)
Borchardt et al (1993)

Biavasco et al (1988) 
Tableau I. Suite.

\begin{tabular}{|c|c|c|c|c|c|c|}
\hline $\begin{array}{l}\text { Organisme } \\
\text { et enzyme }\end{array}$ & $\begin{array}{l}\text { Gène } \\
\text { cloné }\end{array}$ & $\begin{array}{l}M M \\
(k D a)\end{array}$ & $\begin{array}{l}\text { Séquence } \\
\text { signal }\end{array}$ & $\begin{array}{l}\text { Structure } \\
\text { Similarité de séquence }\end{array}$ & Mutation du gène & Références \\
\hline
\end{tabular}

\section{Enzyme}

bifonctionnelle Atl

Amidase-

glucosaminidase

oui

137,5

$(62+51$

(après

maturation)

\section{Clostridium acetobutylicum}

Muramidase LycC

oui

34,9

non

$\mathrm{N}$-terminal :

- domaine catalytique ;

- homologies avec autres muramidase de

Streptomyces globisporus, des phages

de $S$ pneumoniae, du phase mv1

de Lactobacillus bulgaricus

C-terminal :

-5 séquences répétées (23 aa) ;

- homologies avec muramoyl-

pentapeptide carboxypeptidase

de Streptomyces albus
Oshida et al (1995)

Croux et Garcia (1991)

Garcia et al (1988b)

Béliveau et al (1991)

\section{Streptococcus faecalis}

Autolysine

oui

74

(Muramidase?) non $\quad 115$

oui

$\mathrm{N}$-terminal

domaine catalytique

C-terminal :

5 séquences répétées ( 44 aa) 
nombre variable (deux à six). Dans le cas de $S$ pneumoniae, ce domaine serait responsable de la liaison spécifique à la choline présente dans les acides teichoïques de sa paroi (Briese et Hackenbeck, 1985). Des similarités de séquences ont été trouvées soit au niveau du domaine catalytique, soit au niveau du domaine de liaison à la paroi entre des peptidoglycane hydrolases provenant de différentes bactéries ou encore d'origine phagique (tableau I). Parmi ces enzymes, certaines d'entre elles possèdent des caractéristiques particulières. La muramidase M1 de E hirae possède la particularité rare parmi les protéines bactériennes d'être glycosylèe (Kawamura et Shockman, 1983) et de plus, une douzaine de nucléotides sont fixés de manière covalente sur la protéine (Dolinger et al, 1988). Citons également l'enzyme bifonctionnelle de $S$ aureus codée par un gène unique, qui après maturation donne une amidase et une glucosaminidase (Oshida et al, 1995).

\section{Localisation cellulaire}

Afin d'atteindre leur substrat, les autolysines doivent nécessairement être exportées à travers la membrane plasmique. La séquence d'un certain nombre d'entre elles renferme effectivemment une séquence signal potentielle (tableau I). C'est le cas de l'amidase $C$ wIB de $B$ subtilis dont la localisation a par ailleurs été étudiée par immunocytochimie en microscopie électronique (Hobot et Rogers, 1991). Il a été confirmé qu'elle est présente au niveau de la paroi avec un marquage plus important au niveau du septum de cellules en cours de division. Un marquage au niveau du cytoplasme a également été observé. En revanche, pour certaines autolysines telles que l'amidase de $S$ pneumoniae, aucune séquence signal n'a pu être identifiée, ce qui suggère un mode d'exportation non conventionnel. En effet, des expériences d'immunocytochimie après expression de la protéine chez E coli ont montré que la protéine était exportée dans le périplasme, et chez $S$ pneumoniae les expériences ont montré que l'enzyme est localisée au niveau du septum (Diaz et al, 1989). Après exportation, l'amidase semble liée à la surface externe de la membrane par une interaction spécifique avec l'acide lipoteichoïque (LTA). De même, d'autres autolysines ne possèdent pas de séquence signal, l'amidase $\mathrm{CwIC}$ de $B$ subtilis, la muramidase LycC de Clostridium acetobutylicum. Notons que dans le cas de $S$ aureus, l'enzyme LytA initialement identifiée comme une autolysine sans séquence signal s'est révélée être codée par le génome d'un prophage intégré dans le chromosome bactérien (Borchardt et al, 1993).

De manière générale, aucune séquence d'ancrage dans la membrane plasmique n'a été identifiée chez les autolysines séquencées et mentionnées dans le tableau I. En revanche, comme décrit dans le paragraphe précédent, elles semblent contenir un domaine de liaison à la paroi et peuvent être liées soit au peptidoglycane, soit aux acides teichoïques comme l'amidase de $S$ pneumoniae. Notons que certaines autolysines ont été identifiées dans le milieu extracellulaire comme une muramidase de $C$ acetobutylicum (Croux et al, 1992) ou encore l'amidase et la glucosaminidase de $S$ aureus codée par le gène unique at (Oshida et al, 1995). Dans le cas de E hirae, une partie de l'activité de la muramidase M2 est retrouvée dans le surnageant de culture, l'autre partie étant associée aux parois (Kariyama et Shockman, 1992), alors que l'activité de la muramidase M1 est associée en quasi totalité aux cellules.

\section{Régulation et contrôle de l'activité}

Les autolysines sont des enzymes potentiellement dangereuses pour la cellule, ce qui laisse supposer que leur activité est 
extrêmement bien régulée et coordonnée avec les activités impliquées dans la biosynthèse du peptidoglycane. De manière générale, les mécanismes de régulation de l'activité des autolysines ne sont pas parfaitement identifiés. Nous passerons rapidement en revue les différents mécanismes identifiés chez diverses bactéries.

La muramidase M1 de E hirae est synthétisée sous forme d'un zymogène de 130 $\mathrm{kDa}$ exporté vers la paroi puis clivé en une forme active de $87 \mathrm{kDa}$ (Shockman, 1992). Chez $B$ subtilis au contraire, l'action de protéases extracellulaires inactivent une (ou des) autolysine(s) impliquée(s) dans le renouvellement du peptidoglycane (Jolliffe et al, 1980).

Une protéine modificatrice (appelée CwbA) de l'activité de l'amidase CwIB de $B$ subtilis, dont le gène est localisé dans le même opéron que celui de l'amidase, a été décrite (Lazarevic et al, 1992 ; Kuroda et al, 1992). Cette protéine est capable de se lier de façon stoechiométrique avec l'amidase et de stimuler son activité d'un facteur deux ou trois et de plus, de changer son mode d'action d'hydrolyse du peptidoglycane en passant d'un mode d'action aléatoire à un mode d'action séquentiel (Herbold et Glaser, 1975).

L'interaction de l'enzyme avec différents composés de la paroi peut également moduler son activité. Ainsi, l'amidase de $S$ pneumoniae est synthétisée sous une forme non active (forme E) qui est présente uniquement dans le cytoplasme. L'activation de ce précurseur résulterait de la formation d'un complexe avec la choline présente dans les acides teichoïques (Tomasz et Westphal, 1971). Par ailleurs, il a été montré que les acides lipoteichoïques ou des phospholipides tels que la cardiolipine peuvent inhiber l'activité de certaines autolysines comme dans le cas de la muramidase de $E$ hirae. Une modification chimique du substrat telle qu'une $\mathrm{O}$ - ou une $\mathrm{N}$-acétylation peut également influer sur l'activité enzy- matique de la muramidase $\mathrm{M} 1$ de $E$ hirae (Kariyama et Shockman, 1992). Dans la même étude, les auteurs ont mis en évidence l'existence d'un nombre limité de sites de haute affinité de fixation de la muramidase M2 de $E$ hirae.

La localisation de l'enzyme par rapport à son substrat ou encore sa conformation pourrait être un autre facteur intervenant dans la régulation de l'activité. Enfin, la force proton-motrice pourrait également jouer un rôle (Doyle et Koch, 1987).

\section{Rôle physiologique}

Différentes hypothèses ont été avancées concernant le rôle des autolysines dans la physiologie bactérienne, certaines d'entre elles s'appuyant sur des preuves expérimentales indirectes. Les rôles proposés incluaient une intervention dans les processus suivants : 1) la croissance cellulaire en réalisant des coupures au sein du peptidoglycane pour permettre l'insertion de matériel nouvellement synthétisé, 2) la séparation des cellules en fin de division, 3 ) le renouvellement du peptidoglycane et son recyclage, 4) la morphogenèse des flagelles, 5) la transformation de cellules compétentes, 6) la formation et la germination des spores (Rogers et al, 1980).

Actuellement, il existe des preuves directes qui confirment l'intervention des autolysines dans certaines des fonctions énoncées ci-dessus. En effet, après clonage de leur gène de structure, le rôle de certaines autolysines a pu être appréhendé en étudiant des mutants déficients en autolysine obtenus par disruption du gène (tableau I). Ainsi, il a été montré que chez $S$ pneumoniae, l'absence de l'amidase majeure LytA se traduit par la formation de chaînettes de six à huit cellules (au lieu de diplocoques), une absence de lyse en phase stationnaire et une réponse tolérante aux $\beta$ lactames (Sanchez-Puelles et al, 1986). En 
revanche, le mutant déficient en amidase LytA possède une vitesse de croissance normale et peut être transformé normallement. Chez $B$ subtilis, des mutants déficients en chacune des autolysines clonées ont été obtenus. II a été montré que l'absence de l'amidase majeure CwIB se traduit par une résistance accrue à la lyse, une différence dans le taux de renouvellement du peptidoglycane et la motilité flagellaire (Kuroda et Sekiguchi, 1991 ; Margot et Karamata, 1992). En revanche, aucun effet n'a été mis en évidence sur la vitesse de croissance, la morphologie des cellules, leur séparation, leur transformabilité ou la sporulation. L'amidase CwIC apparaît quant à elle induite au cours de la sporulation. Cependant, l'absence de CwIC dans la cellule n'entraîne aucun effet sur la lyse des cellules mères, ni sur la germination des spores, ni sur d'autres caractéristiques des spores (Kuroda et Sekiguchi, 1993). En ce qui concerne l'amidase CwIA (Kuroda et Sekiguchi, 1990) ou la glucosaminidase LytD (Margot et al, 1994), leur inactivation n'entraîne aucun changement des propriétés examinées relatives à la croissance, la motilité flagellaire, la séparation des cellules, l'autolyse, le renouvellement du peptidoglycane, la compétence ou encore la sporulation.

Il apparaît donc que l'étude des mutants n'a répondu jusqu'à maintenant que partiellement à la question du rôle physiologique des autolysines. II est en particulier remarquable que l'absence d'une activité autolysine majeure n'a aucun effet sur la croissance cellulaire. Une explication probable est la présence simultanée de plusieurs autolysines de même spécificité dans la cellule dans de nombreux cas tels ceux de $B$ subtilis, $E$ hirae ou $S$ pneumoniae et l'absence de l'une pourrait être complémentée par la présence d'une autre. Cela a été démontré récemment dans le cas des amidases CwIB et CwIC de B subtilis (Smith et Forster, 1995). II semble par ailleurs qu'un niveau faible d'activité autolysine soit suffi- sant pour la survie et la croissance bactérienne. L'obtention de mutants pluri-déficients en autolysines apparaît nécessaire afin de déterminer si ces autolysines jouent un rôle essentiel dans la cellule.

\section{AUTOLYSINES DES BACTÉRIES LACTIQUES}

\section{Propriétés autolytiques des bactéries lactiques}

\section{Lyse spontanée sur milieu de culture}

L'autolyse de différentes souches de lactocoques (Lactococcus lactis subsp cremoris ou subsp lactis ou encore $L$ lactis subsp lactis biovar diacetylactis) en fin de culture sur milieu liquide synthétique a été étudiée (Vegarud et al, 1983 ; Langsrud et al, 1987). Ces expériences ont permis de mettre en évidence, d'une part des différences entre souches, et d'autre part l'influence de la composition du milieu de culture. En particulier, lorsque le lactose est remplacé par le glucose, l'autolyse est plus rapide et atteint une proportion de cellules plus grande. L'influence de la source de carbone du milieu de culture sur la capacité des cellules à se lyser en fin de croissance a également été décrite dans le cas de $L$ lactis subsp lactis biovar diacetylactis (Moustafa et Collins, 1968). Les cellules de la souche $\mathrm{DRCl}$ cultivées sur un milieu qui contient du glucose sont osmotiquement plus fragiles et se lysent immédiatement après l'arrèt de la croissance (sous l'action du chloramphénicol ou après épuisement du glucose). Selon les auteurs, la fragilité des cellules résulterait de leur incapacité à utiliser le glucose comme précurseur de la galactosamine qui entre dans la composition des acides teichoïques. En conséquence, les cellules présenteraient une paroi moins solide, une fragilité osmotique plus grande 
et une susceptibilité plus grande à l'action des autolysines.

Dans le cas de Streptococcus thermophilus, deux études ont rapporté l'existence de souches qui après une croissance exponentielle rapide sur milieu synthétique, s'autolysent très rapidement (Sandholm et Sarimo, 1981 ; Thomas et Crow, 1983). La susceptibilité à la lyse de ces souches est variable selon la température de croissance et la nature de la source de carbone. Lorsque les bactéries sont cultivées sur milieu contenant du sucrose, elles deviennent résistantes à la lyse (Sandholm et Sarimo, 1981). Dans le cas de la souche NCDO 573, l'autolyse est observée seulement si le $\mathrm{pH}$ final est supérieur à 5 lorsque le disaccharide est épuisé (Thomas et Crow, 1983).

\section{Lyse induite par transfert des cellules en solution tamponnée}

L'autolyse peut être induite en transférant des cellules en culture dans une solution tamponnée, qui vont alors se trouver en situation de privation nutritionnelle. II est possible de cette manière d'étudier l'influence de différents paramètres sur la capacité des cellules à s'autolyser.

Deux études ont recherché les conditions qui favorisent l'autolyse des souches de L lactis subsp cremoris (Mou et al, 1976 ; Niskasaari, 1989). De cette manière, il a été montré que l'autolyse était optimale lorsque les cellules étaient récoltées en phase exponentielle de croissance et plus précisémment juste avant le passage en phase stationnaire. La même observation a été réalisée chez Lactobacillus acidophilus (Coyette et Shockman, 1973), Lactobacillus helveticus (Lortal et al, 1989) ou encore Lactobacillus casei (El Soda et al, 1995).

Ces différentes études ont mis en évidence l'effet de la température, du $\mathrm{pH}$, de la concentration de différents cations et recherché les conditions optimales de lyse. L'influence d'autres composés a également été étudiée. Ainsi, dans le cas des lactocoques, l'autolyse de parois est activée par la trypsine, suggérant soit l'activation d'une autolysine par protéolyse, soit le relargage d'une autolysine dans le milieu de culture permettant une action plus rapide (Niskasaari, 1989). En revanche, l'acide lipotéichoïque et la cardiolipine, un phospholipide, ont un effet inhibiteur sur la lyse comme rapporté dans le cas de $E$ hirae (Cleveland et al, 1976). Chez $L b$ helveticus, Lortal et al (1989) ont observé une stimulation de l'autolyse par l'EDTA ainsi que par les inhibiteurs de la trypsine et de la pepsine, suggérant que dans ce cas, des protéases du lactobacille ont la capacité de dégrader des enzymes autolytiques et donc de les inactiver. Dans le cas de $L b$ acidophilus, un groupement sulfhydryl est nécessaire pour l'autolyse. En effet, les métaux lourds et les agents qui modifient les groupements - $\mathrm{SH}$ libres inhibent la lyse. L'EDTA stimule l'autolyse des cellules mais pas celle des parois (Coyette et Shockman, 1973). Dans ce cas, il est difficile de conclure s'il s'agit d'un effet direct sur l'activité d'une autolysine ou plutôt d'un effet indirect sur la stabilité de l'enveloppe cellulaire. Notons que de manière générale, l'autolyse en solution tamponnée observée dans le cas des lactobacilles est supérieure à celle observée pour les lactocoques.

Dans le cas des lactocoques, ce type d'expérience a permis de montrer que des variants filamenteux de souches de $L$ lactis subsp lactis ou cremoris s'autolysaient moins bien que les cellules normales. Ces observations ont suggéré que la formation de longues chaînes de cellules résultait d'une activité autolysine plus faible et que cette dernière était impliquée dans la séparation des cellules en fin de division (McDonald, 1971). 


\section{Caractérisation des autolysines}

\section{Identification de la spécificité}

L'action d'une glycosidase (muramidase ou glucosaminidase) sur le peptidoglycane entraîne l'apparition de groupements réducteurs, tandis que l'action d'une amidase ou d'une peptidase se traduit par une augmentation des groupements aminés libres (Ghuysen et al, 1966). Il est possible également de distinguer une glucosaminidase (hydrolysant entre Mur et NAcGlc) et une muramidase (hydrolysant entre NAcGlc et Mur) en analysant plus précisément la nature du groupement libéré. Ainsi, en analysant l'autolyse d'une préparation de parois, il est possible d'identifier la nature des enzymes mises en jeu. De cette manière, il a été montré que l'autolysine majeure présente dans des parois de $L$ lactis subsp cremoris est une muramidase (Mou et al, 1976). En revanche, aucune activité amidase ou peptidase n'a été mise en évidence. De même, l'activité autolytique majeure présente dans des parois de $L b$ helveticus (Lortal et al, 1991) et $L b$ acidophilus (Coyette et Ghuysen, 1970) est de type muramidase.

\section{Détection des activités autolysines en gel d'électrophorèse renaturante}

La technique d'électrophorèse renaturante consiste en une électrophorèse SDS-PAGE dans un gel qui contient un substrat d'autolysine tel que des bactéries ou des parois autoclavées. Après migration, le gel est lavé pour éliminer le SDS et les protéines sont renaturées dans un tampon qui contient du Triton X100. La présence d'une autolysine se traduit par l'apparition d'une bande translucide sur un fond opaque qui peut être coloré au bleu de méthylène (Potvin et al, 1988 ; Leclerc et Asselin, 1989).

Nous avons ainsi analysé et comparé le contenu en autolysines d'une quinzaine de souches de lactocoques. Les activités ont été testées sur substrat lactocoques ou microcoques soit dans un extrait cellulaire, soit dans le surnageant de culture. Un profil d'activité semblable a été trouvé sur substrat microcoques pour la plupart des souches testées, y compris pour les souches curées de leurs plasmides $L$ lactis subsp lactis IL1403 et MG1363. Ce profil est constitué par une bande majeure B1 de $45 \mathrm{kDa}$ et de bandes mineures $\mathrm{B} 0, \mathrm{~B} 2$, $\mathrm{B} 3$ de 50,38 et $33 \mathrm{kDa}$ d'intensité variable selon les souches (fig 2A, lignes 1 à 7 et non montré). L'activité majeure est également présente dans le surnageant de culture. Dans le cas de la souche $L$ lactis subsp cremoris AM2 caractérisée comme autolytique au cours de l'affinage (Chapot-Chartier et al, 1994) ainsi que de la souche AM1, aucune bande n'a été détectée dans les mêmes conditions expérimentales (fig 2A, ligne 8). L'enzyme correspondant à la bande $B 1$ paraît cependant présente chez $A M 2$ et peut être révélée en testant l'activité d'une préparation de parois sur substrat microcoques (non montré), mais elle semble en quantité plus faible ou plus difficilement extractible.

Lorsque le contenu en autolysines des mêmes souches est testé sur substrat lactocoques, les profils obtenus sont plus variables (fig $2 \mathrm{~B}$ ) ; une à trois bandes de relativement forte intensité sont détectées au voisinage de $46 \mathrm{kDa}$, celle de plus faible masse moléculaire correspondant à l'activité $B 1$ détectée sur microcoques. Dans le cas des souches $A M 2$ et $A M 1$, une bande majeure $A 2$ de $46 \mathrm{kDa}$ est révélée (fig 2B, ligne 8 ), qui n'est pas active sur microcoques (fig 2A, ligne 8 ).

Cette technique a également permis de mettre en évidence différentes activités chez les lactobacilles. Chez $\mathrm{Lb}$ helveticus, plusieurs activités autolysine ont été révélées sur substrat microcoques ou lactobacilles : une bande de $42 \mathrm{kDa}$ et 6 autres bandes avec des masses moléculaires très voisines, 
comprises entre 29 et $33 \mathrm{kDa}$. Le profil obtenu est identique pour les 29 souches testées. Par ailleurs, en utilisant des parois comme substrat, une bande supplémentaire de $37,5 \mathrm{kDa}$ a pu être mise en évidence (Valence et Lortal, 1995).

Dans le cas de Lb casei, la même tecnique a permis de révéler quatre bandes de masse moléculaire $77,71,55,49 \mathrm{kDa}$ en utilisant soit des cellules entières soit des parois comme substrat, la bande de 55 $\mathrm{kDa}$ étant la bande majeure. De plus, une autre bande de $16 \mathrm{kDa}$ a été détectée en présence de $\mathrm{Mn}^{2+}$ uniquement alors que les autres activitées étaient inhibées ( $F$ Cappa, communication personnelle).

La technique d'électrophorèse renaturante apparaît comme une méthode simple et rapide pour mettre en évidence des activités autolysines et peut également être

\section{A: Microcoques}

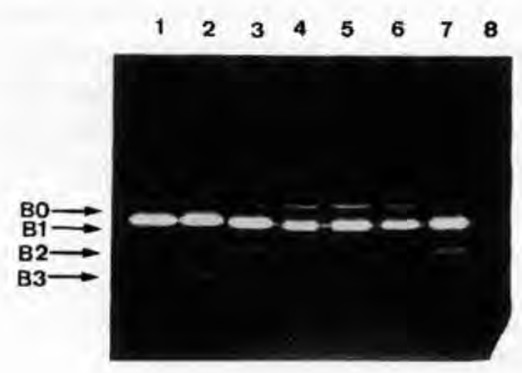

\section{B: Lactocoques}

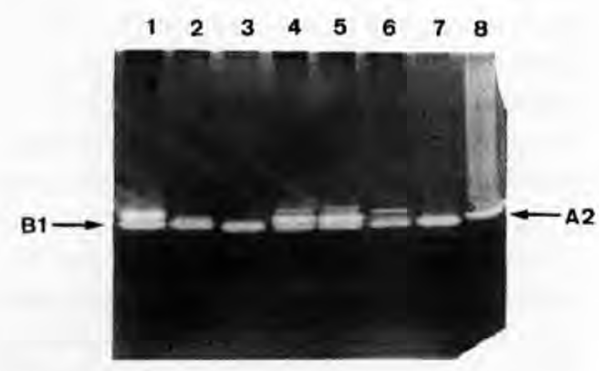

utilisée comme test d'activité au cours d'une purification, par exemple. Cependant, on ne peut exclure l'existence d'autres autolysines chez les bactéries testées. En effet, seules les enzymes capables de se renaturer après un traitement par la chaleur et le SDS peuvent être révélées. De plus, des autolysines composées de plusieurs sousunités non identiques ne peuvent être détectées. Enfin, le nombre de bandes ne reflète pas nécessairement le nombre d'enzymes, une bande mineure pouvant selon sa masse moléculaire représenter soit un produit de dégradation d'une bande majeure soit un précurseur (Leclerc et Asselin, 1989).

Par ailleurs, il faut noter que de nombreuses souches de lactocoques renferment des prophages intégrés dans leur génome et l'on ne peut exclure que cer-

Fig 2. Détection des activités autolysines en électrophorèse renaturante chez différentes souches de Lactococcus lactis subsp cremoris (C13, SK11, US3, E8, AM2) ou subsp lactis (IL1403, MG1363, NCDO763). 1C13 ; 2-IL1403 ; 3- MG1363 ; 4- SK11 ; 5- US3 ; 6E8 ; 7- NCDO763; 8- AM2. L'électrophorèse a été effectuée selon la technique SDS-PAGE (Laemmli, 1970) dans un gel de polyacrylamide à $10 \%$ contenant (A) des microcoques autoclavés $(0,2 \% \mathrm{~m} / \mathrm{v})$ ou (B) des lactocoques autoclavés $(0,4 \% \mathrm{~m} / \mathrm{v})$. Les extraits cellulaires ont été préparés à partir des cellules cultivées sur milieu M17 jusqu'en fin de phase exponentielle de la manière suivante : le culot de cellules a été incubé avec le tampon de dénaturation de Laemmli pendant 3 minutes à $100^{\circ} \mathrm{C}$ puis centrifugé et le surnageant a été récupéré et chargé sur le gel d'électrophorèse.

Detection of autolysin activities by renaturing electrophoresis in different strains of Lactococcus lactis subsp cremoris (C13, SK11, US3, E8, AM2) or subsp lactis (IL 1403, MG1363, NCDO763). 1-C13; 2: IL1403; 3: MG1363; 4: SK11; 5: US3; 6: E8; 7: NCDO763; 8: AM2. SDS-PAGE electrophoresis was performed according to Laemmli (1970) in $10 \%$ polyacrylamide gel containing $(\boldsymbol{A})$ autoclaved micrococci $(0.2 \% \mathrm{w} / \mathrm{V})$ or $(\boldsymbol{B})$ autoclaved lactococci $(0.4 \% \mathrm{w} / \mathrm{v})$. Cellular extracts were prepared from cells grown on $M 17$ medium up to the end of exponential phase in the following way: the cell pellet was incubated in Laemmli denaturation buffer for $3 \mathrm{~min}$ at $100^{\circ} \mathrm{C}$ then centrifuged and the supernatant was recovered and loaded on the electrophoresis gel. 
taines des activités révélées correspondent à des lysines codées par ces prophages.

\section{Clonage du gène codant pour l'autolysine majeure de $L$ lactis}

Le gène codant pour l'autolysine majeure de $L$ lactis a été récemment cloné en criblant une banque d'ADN de lactocoque chez E coli par un test d'activité sur boîte de Petri (Buist et al, 1995). Ce test consiste à inclure des microcoques autoclavés dans le milieu de culture et à rechercher l'apparition de halos de lyse autour des colonies transformantes. De cette manière, le gène codant pour une autolysine nommée AcmA de 46,5 $\mathrm{kDa}$ a été isolé. Une séquence signal de 57 acides aminés a été identifiée. Cette autolysine correspond à l'enzyme majeure (B1) détectée en électrophorèse renaturante. La région $\mathrm{N}$-terminale de la protéine mature possède $60 \%$ d'identité avec la région $N$ terminale de la muramidase-2 d'E hirae (Chu et al, 1992) et l'autolysine de Streptococcus faecalis (Béliveau et al, 1991). Comme les deux autres enzymes, $\mathrm{AcmA}$ contient des séquences répétées du côté C-terminal. Compte tenu des similarités de séquences et des résultats concernant l'identification de l'activité mise en jeu lors de l'autolyse de parois (Mou et al, 1976), AcmA est vraisemblablement une muramidase.

Un mutant délété dans le gène acmA a été construit par la technique de remplacement de gène. Ce mutant forme de longues chaînettes lors de sa croissance, ce qui indique que cette autolysine est impliquée dans la séparation des cellules en fin de division. Par ailleurs, après inactivation du gène, toutes les activités détectées en électrophorèse renaturante disparaissent indiquant qu'elles proviennent toutes de AcmA. Ainsi l'activité $\mathrm{BO}$ décrite ci-dessus représenterait un précurseur de l'autolysine majeure B1, tandis que les activités B2 et B3 correspondraient à des produits de dégradation.

\section{Obtention de souches autolytiques}

Quelques tentatives ont été réalisées afin de sélectionner ou de construire par les techniques du génie génétique des souches autolytiques. Des mutants de lactocoques à lyse thermo-inductible ont été isolés (Feirtag et McKay, 1987a, b). Ces souches sont capables de se lyser lors d'un choc thermique de $30^{\circ} \mathrm{C}$ à $40^{\circ} \mathrm{C}$ qui reproduit l'augmentation de température imposée au cours de la fabrication du Cheddar. Les mutants de $L$ lactis subsp lactis ont été obtenus par mutagenèse chimique. Dans le cas de $L$ lactis subsp cremoris, il s'agissait de souches naturellement thermolytiques, et les auteurs ont émis l'hypothèse que la lyse résultait de l'induction d'un prophage lors du choc thermique. La seconde tentative a été réalisée en exprimant un gène codant pour une lysine de bactériophage chez plusieurs souches de $L$ lactis (Shearman et al, 1992). La croissance des souches obtenues n'était pas affectée pendant la phase exponentielle. En revanche, ces souches se lysaient après avoir atteint la phase stationnaire. Dans ces deux cas, les souches isolées n'ont pas été testées en fabrication fromagère.

\section{AUTOLYSE DANS LE FROMAGE ET INFLUENCE DE LA LYSE SUR L'AFFINAGE}

Des études déjà anciennes ont suggéré et mis en évidence l'importance de la lyse sur la protéolyse au cours de l'affinage (Bie et Sjörström, 1975a,b). Plus récemment, d'autres études se sont attachées à évaluer de manière plus précise, d'une part la capacité des bactéries lactiques du levain à se lyser dans le fromage, et d'autre part l'influence de la lyse sur l'affinage. Les difficultés rencontrées dans ce type d'étude résident dans le choix d'une méthode pour suivre la lyse dans un milieu complexe tel 
que le fromage. Le suivi de la lyse a été réalisé en estimant la viabilité des bactéries, en mesurant la libération de marqueurs intracellulaires tels que des activités enzymatiques ou de l'ADN, ou encore en observant les bactéries en microscopie électronique. Le degré d'affinage a été évalué en quantifiant la protéolyse et par analyse sensorielle.

La capacité de quelques souches de lactocoques à s'autolyser dans le fromage et les conséquences sur l'affinage ont été étudiées dans des fromages de type SaintPaulin (Chapot-Chartier et al, 1994) ou Cheddar (Wilkinson et al, 1994). Dans les deux cas, il a été montré que la capacité des souches de lactocoques à s'autolyser au cours de l'affinage était variable. Dans les fromages de type Saint-Paulin, la souche L lactis subsp cremoris AM2 apparaît lysée dès la première semaine d'affinage comme le montrent les observations en microscopie électronique. La lyse des bactéries s'accompagne d'une décroissance rapide de la viabilité, et les peptidases intracellulaires choisies comme marqueurs de lyse sont présentes dans un extrait de fromage. En revanche, la souche $L$ lactis subsp lactis NCDO763 n'apparaît pas lysée jusqu'à 3 mois d'affinage. Par ailleurs, la lyse de la souche AM2 est accompagnée par un niveau d'acides aminés libres et de petits peptides plus élevé de $30 \%$ à deux mois d'affinage par rapport à la souche NCDO763. Dans le cas du Cheddar, des résultats similaires ont été trouvés quant à la variabilité des souches de lactocoques concernant leur capacité à se lyser d'une part et la corrélation entre lyse et protéolyse plus poussée d'autre part. Dans ce cas, les auteurs ont montré que la lyse entraîne la formation d'une quantité plus élevée d'acides aminés libres et de peptides de faible masse moléculaire. De plus, la lyse peut être corrélée avec une absence d'amertume dans le fromage après 4 mois d'affinage.

Une autre approche a également permis de montrer l'influence de la lyse des bac- téries du levain sur l'affinage. Des fabrications fromagères ont été réalisées avec une souche de lactocoque en présence de bactériophages qui infectent cette souche à des niveaux qui permettent la croissance bactérienne et l'acidification du lait mais entrainent la lyse ultérieure des bactéries. De cette manière, il est possible de s'affranchir des différences entre souches au niveau de leur susceptibilité à l'autolyse ou encore de leur contenu enzymatique et de la stabilité des enzymes. Cette approche initialement utilisée par Lowrie et al (1974) avec une souche amère dans le Cheddar avait permis de montrer que la présence de bactériophages permettait de limiter le développement de l'amertume à un niveau équivalent à celui des souches définies comme non amères en technologie du Cheddar et d'améliorer la flaveur. Récemment, de cette manière, Crow et al (1995) ont étudié l'influence de la lyse de la souche amère $L$ lactis subsp lactis ML8 sur la protéolyse et les qualités organoleptiques. Ces expériences ont montré clairement que la lyse s'accompagne d'une vitesse de protéolyse plus élevée. Lorsque le niveau de phages augmente, la vitesse de formation des acides aminés libres peut être multipliée par un facteur allant jusqu'à 4 . De plus, les auteurs ont observé une absence d'amertume dans les fromages où les bactéries sont lysées. Nous avons obtenu des résultats similaires en lysant la souche NCDO763 non autolytique avec des phages dans des fromages de type Saint-Paulin (Kawabata et al, résultats non publiés). Dans ce cas également, le niveau de lyse peut être corrélé avec la quantité d'acides aminés libres présente dans les fromages, à différents temps d'affinage; l'augmentation de la quantité d'acides aminés libres peut atteindre un facteur 6 après 2 mois d'affinage.

En conclusion, ces différentes études mettent en évidence que la lyse des lactocoques du levain apparaît comme le facteur limitant de la protéolyse. Dans le cas 
du Cheddar, l'intensification de la protéolyse s'accompagne d'une réduction de l'amertume. II faut noter que dans tous les cas rapportés ci-dessus la lyse a été observée en début d'affinage. Par ailleurs, ces résultats suggèrent que certaines peptidases libérées dans le fromage sont relativement stables et permettent une augmentation progressive de la quantité d'acides aminés libres tout au long de l'affinage.

Actuellement, les études effectuées concernent uniquement les lactocoques dans des fromages à pâte pressée et il n'existe pas d'informations disponibles dans les autres cas, en particulier sur la lyse des bactéries lactiques thermophiles présentes dans les fromages à pâte cuite.

\section{PERSPECTIVES}

Le développement des connaissances concernant le système autolytique des bactéries lactiques, la caractérisation moléculaire des enzymes, l'étude de l'influence de divers facteurs physico-chimiques sur leur activité et l'étude de la régulation de l'expression de leur activité devraient fournir une aide précieuse pour contrôler la lyse des bactéries et obtenir des souches qui se lysent précocement au cours de l'affinage.

Différentes approches peuvent actuellement être envisagées pour obtenir des souches autolytiques. La première consiste à sélectionner des souches naturelles, ce qui nécessite l'étude de l'autolyse et des mécanismes mis en jeu dans des conditions physico-chimiques proches de celles du fromage. La deuxième approche consiste à construire des souches pourvues d'un système de lyse inductible par les techniques du génie génétique qui permettent actuellement d'obtenir des souches alimentaires chez différents types de bactéries lactiques (Biswas et al, 1993). Ces souches pourront être construites en plaçant le gène codant pour une autolysine sous le contrôle d'un promoteur inductible par des facteurs physico-chimiques compatibles avec la technologie.

Ces souches permettront en retour d'évaluer de façon plus approfondie et plus précise l'influence de la lyse sur l'affinage, en particulier sur les qualités organoleptiques et la formation d'arômes, ainsi que le moment optimal pour le déclenchement de la lyse. II sera également possible de rechercher ou construire des souches autolytiques qui soient sélectionnées ou améliorées par ailleurs pour d'autres caractères d'intérêt technologique.

\section{REMERCIEMENTS}

Je tiens à remercier $E$ Van Gemert qui a réalisé les électrophorèses, F Cappa (Universita Cattolica, Piacenza) de m'avoir communiqué ses résultats avant publication, JC Gripon pour la lecture critique de ce manuscrit, ainsi que J Gallé et $\mathrm{P}$ Régent pour l'aide à la réalisation des illustrations.

\section{RÉFÉRENCES}

Béliveau C, Potvin C. Trudel J, Asselin A, Bellemare G (1991) Cloning, sequencing, and expresion in Escherichia coli of a Streptococcus faecalis autolysin. $J$ Bacteriol 173, 5619-5623

Biavasco F, Pruzzo C, Thomas C (1988) Cloning and expression of the Staphylococcus aureus glucosaminidase gene in Escherichia coli. FEMS Microbiol Lett 49, 137-142

Bie R, Sjöström G (1975 a) Autolytic properties of some lactic acid bacteria used in cheese production. Part I: Material and methods. Milchwissenschaft $30,653-$ 657

Bie R, Sjöström G (1975 b) Autolytic properties of some lactic acid bacteria used in cheese production. Part II: Experiments with fluid substrates and cheeses. Milchwissenschaft 30, 739-747

Biswas I, Gruss A, Ehrlich SD, Maguin E (1993) High efficiency gene inactivation and replacement system for gram positive bacteria. J Bacteriol 175, 3628 3635

Borchardt SA, Babwah AV, Jayaswal RK (1993) Sequence analysis of the region downstream from a peptidoglycan hydrolase-encoding gene from Sta- 
phylococcus aureus NCTC8325. Gene 137, 253 258

Briese T, Hackenbeck R (1985) Interaction of the pneumococcal amidase with lipoteichoic acid and choline. Eur J Biochem 146, 417-427

Buist G, Kok J, Leenhouts KJ, Dabrowska M, Venema G, Haandrickman AJ (1995) Molecular cloning and nucleotide sequence of the gene encoding the major peptidoglycan hydrolase of Lactococcus lactis, a muramidase needed for cell separation. J Bacteriol $177,1554-1563$

Chapot-Chartier MP. Deniel C, Rousseau M, Vassal L, Gripon JC (1994) Autolysis of two strains of Lactococcus lactis during cheese ripening. Int Dairy $\mathrm{J} 4$, 251-269

Chu CP, Kariyama R, Daneo-Moore L, Shockman GD (1992) Cloning and sequence analysis of the muramidase-2 gene from Enterococcus hirae. $J$ Bacteriol 174, 1619-1625

Cleveland RF, Daneo-Moore L, Wicken AJ, Shockman GD (1976) Effect of lipoteichoic acid and lipids on lysis of intact cells of Streptococcus faecalis. $J$ Bac. teriol 127, 1582-1584

Coyette J, Ghuysen JM (1970) Wall autolysin of Lactobacillus acidophilus strain 63 AM Gasser. Biochemistry 9, 2952-2955

Coyette J, Shockman GD (1973) Some properties of the autolytic $\mathrm{N}$-acetylmuramidase of Lactobacillus acidophilus. J Bacteriol 114, 34-41

Croux C, Garcia J-L (1991) Sequence of the lyc gene encoding the autolytic lysozyme of Clostridium acetobutylicum ATCC824: comparison with other lytic enzymes. Gene 104, 25-31

Croux C, Canard B, Goma G, Soucaille P (1992) Purification and characterization of an extracellular muramidase of Clostridium acetobutylicum ATCC824 that acts on non- $\mathrm{N}$-acetylated peptidoglycan. Appl Environ Microbiol 58, 1075-1081

Crow VL, Martley FG, Coolbear T, Roundhill SJ (1995) The influence of phage assisted lysis of Lactococcus lactis subsp lactis ML 8 on Cheddar cheese ripening. Int Dairy J 5, 451-472

Diaz E, Garcia E, Ascaso C, Mendez E, Lopez R, Garcia JL (1989) Subcellular localization of the major pneumococcal autolysin: a peculiar mechanism of secretion in Escherichia coli. J Biol Chem 264, 1238-1244

Dolinger DL, Schramm VL, Shockman GD (1988) Covalent modification of the $\beta-1,4-N$-acetylmuramoylhydrolase of Streptococcus faecium with 5-mercaptouridine monophosphate. Proc Nat Acad Sci 85 , 6667-6671

Doyle RJ, Koch AL (1987) The functions of autolysins in the growth and division of Bacillus subtilis. CRC Crit Rev Microbiol 15, 169-222

EI Soda M, Farkye N, Vuillemard JC, Simard RE, Olson, NF, El Kholy W, Dako E, Medrano E, Gaber M, Lim $L$ (1995) Autolysis of lactic acid bacteria: impact on flavour development in cheese. In: Food Flavors: Generation, Analysis and Process Influence (G. Charalambous, Ed), Elsevier Science, Amsterdam

Feirtag JM, McKay LL (1987a) Isolation of Streptococcus lactis C2 mutants selected for temperature sensitivity and potential role in cheese manufacture. J Dairy Sci 70, 1773-1778

Feirtag JM, McKay LL (1987b) Thermoinducible lysis of temperature-sensitive Streptococcus cremoris strains. J Dairy Sci 70, 1779-1784

Forster SJ (1991) Cloning, expression, sequence analysis and biochemical characterization of an autolytic amidase of Bacillus subtilis $168 \operatorname{trpC2}$. $J$ Gen Microbiol 137, 1987-1998

Garcia E, Garcia J-L, Ronda C, Garcia P, Lopez R (1985) Cloning and expression of the pneumococcal autolysin gene in Escherichia coli. Mol Gen Genet 201, 225-230

Garcia P, Garcia J-L, Garcia E, Lopez R (1986) Nucleotide sequence and expresion of the pneumococcal autolysin gene from its own promoter in Escherichia coli. Gene 43, 265-272

Garcia E, Garcia JL, Garcia P, Arraras A, Sanchez-Puelles JM, Lopez R (1988a) Molecular evolution of lytic enzymes of Streptococcus pneumoniae and its bacteriophages. Proc Natl Acad Sci USA 85, 914-918

Garcia JL, Garcia E, Sanchez-Puelles JM, Lopez R (1988b) Identification of a lytic enzyme of Clostridium acetobutylicum that degrades choline-containing pneumococcal cell walls. FEMS Microbiol Lett 52, 133-138

Garcia P, Garcia JL, Garcia E, Lopez R (1989) Purification and characterization of the autolytic glycosidase of Streptococcus pneumoniae. Biochem Biophys Res Commun 158, 251-256

Ghuysen JM (1968) Use of bacteriolytic enzymes in determination of wall structure and their role in cell metabolism. Bacteriol Rev 32, 425-464

Ghuysen JM, Tipper DJ, Strominger JL (1966) Enzymes that degrade bacterial cell walls. Meth Enzymol 8 , 685-699

Herbold DR, Glaser L (1975) Bacillus subtilis N-acetylmuramic acid L-alanine amidase. J Biol Chem 250, 1676-1682

Hobot JA, Rogers HJ (1991) Intracellular location of the autolytic the $\mathrm{N}$-acetylmuramoyl-L-alanine amidase in Bacillus subtilis 168 and in an autolysis-deficient mutant in immunoelectron microscopy. J Bacteriol $173,961-967$

Höltje J-V, Tuomanen El (1991) The murein hydrolases of Escherichia coli. properties, functions and impact on the course of infections in vivo. J Gen Microbiol $137,441-454$

Jayaswal R, Lee YI, Wilkinson BJ (1990) Cloning and expresion of a Staphylococcus aureus gene encoding a peptidoglycan hydrolase activity. J Bacteriol 172 , 5783-5788 
Jolliffe LK, Doyle RJ, Streips UN (1980) Extracellular proteases modify cell wall turnover in Bacillus subtilis. J Bacteriol 141, 1199-1208

Kariyama R, Shockman GD (1992) Extracellular and cellular distribution of muramidase-2 and muramidase-1 of Enterococcus hirae ATCC 9790. $J$ Bacteriol 174, 3236-3241

Kawamura T, Shockman GD (1983) Purification and some properties of the endogenous, autolytic $\mathrm{N}$-acetylmuramoylhydrolase of Streptococcus faecium, a bacterial glycoenzyme. J Biol Chem 258, 9514-9521

Kuroda A, Sekiguchi J (1990) Cloning, sequencing and genetic mapping of a Bacillus subtilis cell wall hydrolase gene. J Bacteriol 136, 2209-2216

Kuroda A, Sekiguchi J (1991) Molecular cloning and sequencing of a major Bacillus subtilis autolysin gene. J Bacteriol 173, 7304-7312

Kuroda A, Sekiguchi J (1993) Molecular cloning of a sporulation-specific cell wall hydrolase gene of BacilIus subtilis. J Bacteriol 175, 6260-6268

Kuroda A, Rashid MH, Sekiguchi J (1992) Molecular cloning and sequencing of the upstream region of the major Bacillus subtilis autolysin gene: a modifier protein exhibiting sequence homology to the major autolysin and the spollD product. J Gen Microbiol 138, 1067-1076

Laemmli UK (1970 Cleavage of the structural proteins during the assembly of the head bacteriophage T4. Nature 227, 680-685

Langsrud T, Landaas A, Castberg HB (1987) Autolytic properties of different strains of group $\mathrm{N}$ streptococci. Milchwissenschaft 49, 556-560

Lazarevic V, Margot P, Soldo B, Karamata D (1992) Sequencing and analysis of the Bacillus subtilis IytRABC divergon: a regulatory unit encompassing the structural genes of the $\mathrm{N}$-acetylmuramoyl-L-alanine amidase and its modifier. J Gen Microbiol 138 , 1949-1961

Leclerc D, Asselin A (1989) Detection of bacterial cell wall hydrolases atter denaturing polyacrylamide electrophoresis. Can J Microbiol 35, 749-753

Lopez R, Garcia JL, Garcia E, Ronda C, Garcia P (1992) Structural analysis and biological significance of the cell wall lytic enzymes of Streptococcus pneumoniae and its bacteriophage. FEMS Microbiol Lett $100,439-448$

Lortal S, Boyaval P, Van Heijenoort J (1989) influence de plusieurs facteurs sur Pautolyse de Lactobacillus helveticus CNRZ 414. Lait 69, 223-231

Lortal S, Rousseau M, Boyaval P, Van Heijenoort J (1991) Cell wall and autolytic system of Lactobacillus helveticus ATCC 12046. J Gen Microbiol 137. 549-559

Lowrie RJ, Lawrence RC, Pederby MF (1974) Cheddar cheese flavour. V. Influence of bacteriophage and cooking temperature on cheese made under control- led bacteriological conditions. N J Dairy Sci Technol $9,116-121$

Margot P, Karamata D (1992) Identification of the structural genes for $\mathrm{N}$-acetylmuramoyl- $\mathrm{\text {-alanineamidase }}$ and its modifier in Bacillus subtilis 168: inactivation of these genes by insertional mutagenesis has no effect on growth or cell separation. Mol Gen Genet 232, 359-366

Margot P, Mauël C, Karamata D (1994) The gene of the $\mathrm{N}$-acetylglucosaminidase, a Bacillus subtilis 168 cell wall hydrolase not involved in vegetative cell autolysis. Mol Microbiol 12, 535-545

McDonald IJ (1971) Filamentous forms of Streptococcus cremoris and Streptococcus lactis. Observations on structure and susceptibility to lysis. Can J Microbiol $17,897-902$

Monnet V, Chapot-Chartier M-P, Gripon J-C (1993) Les peptidases des lactocoques. Lait 73, 97-108

Mou L, Sullivan JJ, Jago GR (1976) Autolysis of Streptococcus cremoris. J Dairy Res 43, 275-282

Moustafa HH, Collins EB (1968) Molar yields of certain lactic acid bacteria as influenced by autolysis. $J \mathrm{Bac}$. teriol $96,117-125$

Niskasaari K (1989) Characteristics of the autolysis of variants of Lactococcus lactis subsp cremoris. J Dairy Res 56, 639-649

Olson NF (1990) The impact of lactic acid bacteria on cheese flavor. FEMS Microbiol Rev 87, 131-148

Oshida $T$, Sugai M, Komatsuzawa $H$, Hong YM, Suginaka H, Tomasz A (1995) A Staphylococcus aureus autolysin that has an $\mathrm{N}$-acetylmuramoyl-L-alanine amidase domain and a endo- $\mathrm{B}-\mathrm{N}$-acetylglucosaminidase domain: cloning, sequence analysis and characterization. Proc Nat/ Acad Sci USA 92, 285-289

Potvin C, Leclerc D, Tremblay G, Asselin A, Bellemare G (1988) Cloning, sequencing and expression of a Bacillus bacteriolytic enzyme in Escherichia coli. $\mathrm{Mol}$ Gen Genet 314, 241-248

Pritchard GG, Coolbear T (1993) The physiology and biochemistry of the proteolytic system in the lactic acid bacteria. FEMS Microbiol Rev 12, 179-206

Rogers HJ, Perkins HR, Ward JB (1980) Microbial cell walls and membranes. Chapman and Hall, London

Sanchez-Puelles JM, Ronda C, Garcia JL, Garcia P. Lopez R, Garcia E (1986) Searching for autolysin functions. Characterization of a pneumococcal mutant deleted in the lytA gene. Eur J Biochem 158, 289-293

Sandholm E, Sarimo SS (1981) Autolysis of Streptococcus thermophilus. FEMS Microbiol Lett 11, 125129

Schleifer KH, Kandler O (1972) Peptidoglycan types of bacterial cell walls and their taxonomic implications. Bacteriol Rev 36, 407-477 
Shearman CA, Jury K, Gassin MJ (1992) Autolytic Lactococcus lactis expressing a lactococcal bacteriophage lysin gene. Biotechnology 10, 196-199

Shockman GD (1992) The autolytic ("suicidase") system of Enterococcus hirae: from lysine depletion autolysis to biochemical and molecular studies of the two muramidases of Enterococcus hirae ATCC 9790. FEMS Microbiol Lett 100, 261-268

Shockman GD, Barrett JF (1983) Structure, function and assembly of cell walls of Gram positive bacteria. Annu Rev Microbio/ 37, 501-527

Shockman GD, Höltje JV (1994) Microbial peptidoglycan (murein) hydrolases. In: New Comprehensive Biochemistry. Vol 27. Bacterial Cell Wall (JM Ghuysen and R Hakenbeck Eds) Elsevier, Amsterdam. 131166

Smith TJ, Foster SJ (1995) Characterization of the involvement of two compensatory autolysins in mother cell lysis during sporulation of Bacillus subtilis 168 . J Bacteriol 177, 3855-3862

Tan PST, Chapot-Chartier MP, Pos KM, Rousseau M, Boquien CY, Gripon JC, Konings WN (1992) Localization of peptidases in lactococci. Appl Environ Microbiol 58, 285-290

Thomas TD, Crow VL (1983) Lactose and sucrose utilization by Streptococcus thermophilus. FEMS Microbiol Lett 17, 13-17
Tomasz A, Westphal M (1971) Abnormal autolytic enzyme in a pneumococcus with altered teichoic acid composition. Proc Natl Acad Sci USA 68, 26272630

Valence F, Lortal S (1995) Zymogram and preliminary characterization of Lactobacillus helveticus autolysins. Appl Environ Microbiol, 61, 3391-3399

Vegarud G, Castberg HB, Langsrud T (1983) Autolysis of group $\mathrm{N}$ streptococci. Effects of media composition modifications and temperature J Dairy Sci66, 22942302

Visser FMW (1977) Contribution of enzymes from rennet, starter bacteria and milk to proteolysis and flavour development in Gouda cheese. 2. Development of bitterness and cheese flavour. Neth Milk Dairy J31, 188-209

Visser S (1993) Proteolytic enzymes and their relation to cheese ripening and flavor: an overview. J Dairy Sci 76, 329-350

Wang X, Wilkinson BJ, Jayaswal RK (1991) Sequence analysis of a Staphylococcus aureus gene encoding a peptidoglycan hydrolase activity. Gene 102, 105109

Wilkinson MG, Guinee TP, O'Callaghan DM, Fox PF (1994) Autolysis and proteolysis in different strains of starter bacteria during Cheddar cheese ripening. J Dairy Res 61, 249-262 